\title{
Measuring Hall viscosity of graphene's electron fluid
}

\begin{abstract}
A. I. Berdyugin'1, S. G. Xu ${ }^{1,2}$, F. M. D. Pellegrino ${ }^{3,4}$, R. Krishna Kumar ${ }^{1,2}$, A. Principi ${ }^{1}$, I. Torre ${ }^{5}$, M. Ben Shalom ${ }^{1,2}$, T. Taniguchi ${ }^{6}$, K. Watanabe ${ }^{6}$, I. V. Grigorieva', M. Polini ${ }^{1,7}$, A. K. Geim ${ }^{1,2 *}$, D. A. Bandurin ${ }^{1 *}$

${ }^{1}$ School of Physics and Astronomy, University of Manchester, Manchester M13 9PL, UK. ${ }^{2}$ National Graphene Institute, University of Manchester, Manchester M13 9PL, UK. ${ }^{3}$ Dipartimento di Fisica e Astronomia, Università di Catania, Via S. Sofia, 64, I-95123 Catania, Italy. ${ }^{4}$ INFN, Sez. Catania, I-95123 Catania, Italy. ${ }^{5}$ ICFO-Institut de Ciencies Fotoniques, The Barcelona Institute of Science and Technology, 08860 Castelldefels (Barcelona), Spain. ${ }^{6}$ National Institute for Materials Science, 1-1 Namiki, Tsukuba, 3050044 Japan. ${ }^{7}$ Istituto Italiano di Tecnologia, Graphene Labs, Via Morego 30, 16163 Genova, Italy.
\end{abstract}

*Corresponding author. Email: geim@manchester.ac.uk (A.K.G.); bandurin.d@gmail.com (D.A.B.)

An electrical conductor subjected to a magnetic field exhibits the Hall effect in the presence of current flow. Here we report a qualitative deviation from the standard behavior in electron systems with high viscosity. We find that the viscous electron fluid in graphene responds to non-quantizing magnetic fields by producing an electric field opposite to that generated by the ordinary Hall effect. The viscous contribution is substantial and identified by studying local voltages that arise in the vicinity of currentinjecting contacts. We analyze the anomaly over a wide range of temperatures and carrier densities and extract the Hall viscosity, a dissipationless transport coefficient that was long identified theoretically but remained elusive in experiments.

Electron transport in metallic systems is routinely described in terms of classical charges that whizz through the bulk and scatter at various defects such as impurities, edges, lattice vibrations, etc. This semiclassical picture does not hold however for materials where defects are scarce and electronelectron collisions provide the shortest scattering length. In the latter case electrons respond to external fields collectively so that their transport resembles a classical fluid flow (1-3). The studies of electron hydrodynamics have been hampered by the scarcity of experimental systems in which both impurity and electron-phonon scattering - which do not conserve the electron momentum - are weak so that electron-electron collisions become the dominant source of scattering. The situation has changed recently owing to improvements in materials quality $(4,5)$ including high-quality graphene with its exceptionally weak electron-phonon coupling (6-8). Transport experiments have provided clear evidence for a fluid-like behavior of charge carriers in graphene, which reveals itself in, e.g., negative vicinity resistance $(6,7)$ and superballistic transport (8). Good agreement between the experiments and theory indicates that electron transport in high-quality graphene at temperatures $(T)$ above $100 \mathrm{~K}$ is consistent with the hydrodynamics description (7, 9-13).

So far, studies of electron hydrodynamics focused on zero magnetic field $B$. On the other hand, the finite $B$ regime described by a combination of the Navier-Stokes and Maxwell's equations is relevant for many research fields ranging from astrophysics to plasma physics to geophysics and engineering. For static $B$, the viscous response of a charged fluid is described by a tensor in the Navier-Stokes equation that contains a dissipationless, off-diagonal coefficient called Hall or odd viscosity $v_{\mathrm{H}}$ (14-17). To get some qualitative insight into the behavior of a two-dimensional electron fluid subjected to a non-quantizing magnetic field, we plot the calculated electric potential distribution $\phi(\boldsymbol{r})$ expected near a narrow current injector in zero (Fig. 1A) and finite $B$ (Fig. 1B). The injected current $I$ entrains adjacent fluid regions, which results in negative lobes of the potential near the injector (18, 19). In zero $B$ (Fig. 1A), the lobes are symmetric with respect to the injection direction and, for restricted geometries, can be accompanied by whirlpools of electric current (18-22). Finite $B$ induces considerable asymmetry in $\phi(\boldsymbol{r})$ (Fig. 1B), which involves the following three contributions. First, the ordinary Hall effect (HE) causes the well-known potential difference $V_{\mathrm{H}}=I B / n e$ between the left and right sides of the halfplane (Fig. 1C) where $n$ is the carrier density and $e$ the elementary charge. The second contribution comes from the longitudinal viscosity $v(B)$ and, in small $B$, is practically indistinguishable from that shown in Fig. 1A for zero $B$. The third contribution arises from Hall viscosity, the main subject of our interest here. The $v_{\mathrm{H}}$ contribution (Fig. 1D) is opposite in sign to the classical Hall effect (Fig. 1C); $v_{\mathrm{H}}$ suppresses the normal Hall response but this influence rapidly decays away from the injector region (Fig. 1D). The latter feature makes it difficult to observe the Hall viscosity using conventional devices and measurement geometries such as the standard Hall bar configuration $(15,16)$. As shown below, the vicinity geometry (Fig. 1E) allows us to distinguish between the ordinary $\mathrm{HE}$ and the anomalous one caused by $v_{\mathrm{H}}$.

Our devices were multiterminal Hall bars such as shown in Fig. 1E and figs. S1A-B. They were made from graphene encapsulated between hexagonal boron-nitride crystals (22). The Hall bars had typical widths of $2-4 \mu \mathrm{m}$ and were endowed with narrow $(\sim 0.3 \mu \mathrm{m})$ and closely spaced $(\sim 0.5 \mu \mathrm{m})$ 
voltage probes (Fig. 1E). Such submicrometer probes are essential for detection of viscous effects as seen from the spatial scale of Figs. 1, A-D. Several devices made from mono- and bilayer graphene (MLG and BLG, respectively) were studied, all exhibiting similar behavior. The data reported below are from three MLG and two BLG devices which were studied in great detail. They had typical mobilities exceeding $\sim 100,000$ $\mathrm{cm}^{2} \mathrm{~V}^{-1} \mathrm{~s}^{-1}$ at all $T$ up to $300 \mathrm{~K}(22)$, which ensured micrometer-scale transport with respect to momentum-non-conserving scattering over the entire $T$ range explored in the experiments (fig. S1); for comparison, the electron-electron mean free path at representative $n=10^{12} \mathrm{~cm}^{-2}$ and $T=150 \mathrm{~K}$ is $\sim 0.4 \mu \mathrm{m}$, shorter than a typical distance of $\sim 1 \mu \mathrm{m}$ at which the viscous contribution was probed $(8,11)$.

In the vicinity geometry, the current $I$ is injected through a narrow contact (e.g., probe 1 in Fig. 1E) into a wide graphene channel, and the local potential $\phi$ is measured using probe 3 positioned at the distance $L$ from the injector. Contacts 2 and 4 (chosen sufficiently far away from the injection region) complete the electric circuit, serving as the drain and reference-voltage contacts, respectively. The vicinity resistance is defined as $R_{\mathrm{v}}=R_{34,12}=V_{34} / I_{12}$ where $V_{34}$ is the voltage drop between 3 and 4. As per Figs. 1, A-D, $R_{\mathrm{v}}$ is expected to be sensitive to viscous effects $(6,7,18,22)$. According to the previous experiments and theory $(6,7,12,18), R_{\mathrm{v}}$ is a nonmonotonic function of $T$ such that $R_{\mathrm{v}}$ is positive in the ballistic regime at low $T$, changes its sign to negative with increasing $T$, passes through a minimum and then starts growing. The negative sign of $R_{\mathrm{v}}$ is a clear indicator that electron-electron scattering strongly affects ballistic transport $(6,7,12,18,22)$ whereas the turning point marks the onset of the regime where the hydrodynamics approach becomes applicable ( 6 , 7).

For the purpose of this report, we focus on the latter regime which in our devices starts above 100-150 K, depending on $n$ (fig. S2A). In addition, we set several other constraints on variables used in the experiments. First, we limit ourselves to $B<40 \mathrm{mT}$ such that the cyclotron radius always exceeds our devices' width. This is to avoid hydrodynamics effects to be obscured by those caused by Landau quantization and electron focusing (23). In addition, such small $B$ are not expected to affect the longitudinal component of $v$ (see below). Second, to avoid unrelated effects stemming from thermal excitations and charge inhomogeneity, we carry out experiments away from the charge neutrality point, at $n$ of the order of $10^{12} \mathrm{~cm}^{-2}$. Finally, we employ small $I \leq 1 \mu$ A to stay in the linear response regime and avoid nonlinear effects including electron heating $(2,6)$.

Examples of $R_{\mathrm{v}}(n)$ in the hydrodynamic regime are shown in Fig. 1F for one of our MLG devices. In agreement with the previous studies $(6,7), R_{\mathrm{v}}$ in zero $B$ is negative for all $n$ away from the charge neutrality and is practically symmetric for electron and hole doping (positive and negative $n$, respectively). The small positive field of $20 \mathrm{mT}$ shifts the $R_{\mathrm{v}}$ curves in opposite directions for electrons and holes, as indicated by the green arrows in Fig. $1 \mathrm{~F}$. The shifts are opposite for negative $B$. This behavior implies a contribution that is antisymmetric with respect to $B$ and $n$, similar to the ordinary Hall effect. However, the latter cannot possibly explain the observed shifts because in the vicinity geometry voltage probes are placed on the same side of the current path, which cancels the ordinary HE contribution to the measured voltages. A formal proof of this can be found in (16). Experimentally, we have also checked that there is no ordinary HE contribution for the vicinity geometry using similar graphene devices but exhibiting low mobility (fig. S2, D-F). Furthermore, it is important to compare the sign of the $R_{\mathrm{v}}$ changes induced by $B$ with the sign of the ordinary HE. To keep the same sign convention for $B$ and $n$, it is instructive to measure the local Hall resistance $R_{35,12}$ (Fig. 1E and fig. S6) instead of using the standard Hall geometry. In this case, we use contact 5 instead of 4 and keep all the other contacts same as in the $R_{\mathrm{v}}$ measurements. This swap places the voltage probes at the opposite sides of the current path, giving rise to the voltage drop $V_{\mathrm{H}}$ due to the ordinary HE. The antisymmetric-in- $B$ part of $R_{35,12}$ (to avoid a contribution from longitudinal resistivity) is plotted in Fig. IF (dashed lines). It shows that the ordinary HE induces $\phi$ of the opposite polarity with respect to those causing the $B$-shifts in $R_{\mathrm{v}}$. Indeed, the vicinity curve in Fig. $1 \mathrm{~F}$ is shifted, for example, upwards for hole doping and positive $B$, whereas the ordinary HE would shift it downwards [also, see Section 6 of (22)]. This behavior agrees well with the opposite signs of the contributions expected from $V_{\mathrm{H}}$ and $v_{\mathrm{H}}$ toward $R_{\mathrm{v}}$ as shown in theoretical Fig. 1, C and D.

For further analysis, we define the Hall (odd) component of the vicinity resistance as $R_{\mathrm{A}}(B)=\left[R_{\mathrm{v}}(B)-R_{\mathrm{v}}(-B)\right] / 2$. The antisymmetrization removes the contributions that are symmetric in $B$ and caused by the longitudinal viscosity $v$ and the Ohmic flow $(6,16)$. Figure $2 \mathrm{~A}$ and fig. S3A show examples of the $R_{\mathrm{A}}(B)$ curves for MLG and BLG devices, respectively. Within the ranges of $T$ and $B$ used in our experiment, the dependences are linear in $B$ for all the studied devices and for all $L$. By analogy with the conventional Hall coefficient, where $\alpha_{\mathrm{H}}=R_{\mathrm{H}} n e / B \equiv 1$, it is instructive to introduce the viscous Hall coefficient, $\alpha_{\mathrm{VH}}=R_{\mathrm{A}} n e / B(22)$. In this form, the antisymmetric contribution $R_{\mathrm{A}}$ is effectively normalized by the ordinary HE, which provides a sense of the magnitude for the observed viscous effects.

Figure 2B shows the $T$ dependence of $\alpha_{\mathrm{VH}}$ obtained using data such as those in Fig. 2A. Above $100 \mathrm{~K}$, where the hydrodynamic regime becomes fully developed $(6,7,10,12)$, the viscous contribution reaches $20 \%$ of the ordinary $\mathrm{HE}$ and has the opposite sign (Fig. 2B). $\left|\alpha_{\mathrm{VH}}\right|$ decreases with increasing $T$ and eventually disappears below noise above room $T$. This $T$ 
dependence was found universal for all the studied devices (fig. S2C). Figures2, D and E, details the observed behavior by plotting $\alpha_{\mathrm{VH}}(T, n)$ for MLG and BLG. The maps are somewhat different because of different viscosities of the two graphene systems $(6,10,11)$ but show similar trends as functions of $n$ and $T$. We have also studied how $\alpha_{\mathrm{VH}}$ depends on $L$ and found that it decreases with increasing $L$, practically disappearing if the voltage probe is placed further than $\sim 2 \mu \mathrm{m}$ from the current-injecting contact (Fig. 2C). The latter highlights the importance of the vicinity geometry to detect viscous effects.

The anomalous viscous contribution to the Hall effect, which is found using the vicinity geometry, is fully consistent with our measurements of a local Hall resistance [Section 6 of (22)]. To this end, we again employed voltage contacts close to the current injector (e.g., using $R_{35,16}$ ) and compared those measurements with the standard HE geometry $\left(R_{24,16} \equiv\right.$ $R_{16,24}$ ). The latter exhibited the ordinary HE with $\alpha_{\mathrm{H}}=1$, as expected. In contrast, the local Hall resistance was notably suppressed in the hydrodynamic regime (fig. S6) and agreed quantitatively with the behavior of $\alpha_{\mathrm{VH}}$ reported above.

Let us now turn to theory. In the linear-response and steady-state regimes, two-dimensional viscous transport in the presence of a perpendicular field $B$ (in the $z$ direction) is described by the Navier-Stokes equation

$$
\frac{\sigma_{0}}{n e} \nabla \phi(\boldsymbol{r})=\left(1-D_{v}^{2} \nabla^{2}\right) v(\boldsymbol{r})+\omega_{\mathrm{c}} \tau\left(1+D_{\mathrm{H}}^{2} \nabla^{2}\right) v(\boldsymbol{r}) \times z \text { (1) }
$$

in conjunction with the continuity equation and no-slip boundary conditions $(16,22)$. Here, $v(\boldsymbol{r})$ is the local fluid velocity, $\omega_{c}=e B / m$ the cyclotron frequency for electrons with the effective mass $m, \sigma_{0}=n e^{2} \tau / m$ the Drude conductivity and $\tau$ the transport time with respect to momentum-non-conserving collisions such as, e.g., scattering on phonons. The right-hand side of Eq. 1 contains two terms. The first describes the electric current and viscous friction parameterized through the diffusion constant, $D_{v}=\sqrt{v \tau}$. The second term arises from the Lorentz force $\boldsymbol{F}_{\mathrm{L}}=-\left(\omega_{\mathrm{c}} m\right) v(\boldsymbol{r}) \times z$ and its viscous counterpart that depends on $v_{\mathrm{H}}$ and is parameterized through another diffusion constant, $D_{\mathrm{H}}=\sqrt{v_{\mathrm{H}} / \omega_{\mathrm{c}}}$. Note that the Hall friction acts against $\boldsymbol{F}_{\mathrm{L}}$ which also means that $v_{\mathrm{H}}$ does not perform any work on the electron fluid and, therefore, it is a dissipationless coefficient.

For the half-plane geometry (fair approximation for our devices) and close to the injection point, the above equation can be solved analytically (16) yielding $\phi(\boldsymbol{r})$ shown in Fig. 1, A to D (fig. S4 provides examples of the potential and current maps calculated taking into account the finite device width). The Hall contribution to the vicinity resistance can be written as

$$
R A=-\sigma_{0}^{-1} \xi\left(\frac{L}{D_{v}}\right) \frac{v_{\mathrm{H}}}{v}
$$

where $\xi(x)=\left[L_{1}(x)-I_{1}(x)\right] / 2 x$, and $L_{1}(x)$ and $I_{1}(x)$ are the modified Struve and Bessel functions, respectively (16). The function $\xi\left(L / D_{v}\right)$ decreases monotonically with increasing $L$, behaving as $D_{v} / \pi L$ for $L \gg D_{v}$. The $L$ dependence expected for our devices using Eq. 2 is plotted in Fig. 2C, showing reasonable agreement with the experiment (especially in terms of the absolute values), even without taking into account the finite width $(\sim 0.3 \mu \mathrm{m})$ of our current and voltage contacts.

The measured $R_{\mathrm{A}}(B)$ such as shown in Fig. 2A can be used to extract $v_{\mathrm{H}}$. To this end, we rewrite Eq. 2 as $v_{\mathrm{H}}=-R_{\mathrm{A}} \sigma_{0} v / \xi(L / \sqrt{v \tau})$ where $\sigma_{0}$ and $\tau$ can be deduced from standard longitudinal resistivity measurements $(6,8)$. The longitudinal viscosity $v(B)$ can be approximated using the semiclassical expression $(16,24,25) v(B)=v_{0} \frac{B_{0}^{2}}{B^{2}+B_{0}^{2}}$ where $v_{0}$ is the kinematic viscosity in zero $B$, and $B_{0}=\hbar v_{\mathrm{F}} k_{\mathrm{F}} /\left(8 e v_{0}\right)$ is the characteristic magnetic field expressed through the Fermi wave number $k_{\mathrm{F}}$, the Fermi velocity $v_{\mathrm{F}}$, and the reduced Planck constant $\hbar$. For the reported range of $n$ and $T, B_{0}$ is much larger than the fields in our experiments. Accordingly, we can assume $v \approx v_{0}$ in Eq. 2 and then use $v_{0}$ found experimentally in (8). Employing the above protocol, it is straightforward to calculate $v_{\mathrm{H}}$ and its $B$ and $T$ dependences. Examples are shown in Fig. 3, A and B. One can see that the Hall viscosity is linear in $B$ and rapidly decreases with increasing $T$.

For consistency, we crosschecked the above analysis against the results obtained previously (8) for zero-field vis$\operatorname{cosity} v_{0}$. To this end, we note that the field dependence of $R_{\mathrm{A}}$ $=R_{\mathrm{A}}\left[v_{\mathrm{H}}(B), v(B)\right]$ originates from changes in both longitudinal and Hall viscosities. The full formula for $v(B)$ is given above whereas the same semiclassical consideration $(16,24)$ for the Hall viscosity yields $v_{\mathrm{H}}(B)=v_{0} \frac{B B_{0}}{B^{2}+B_{0}^{2}}$. This allows us to redefine the anomalous Hall contribution as $R_{\mathrm{A}}\left[v_{0}, B\right]$ and calculate $v_{0}$ from the measured $R_{\mathrm{A}}(B)$ dependences such as in Fig. 2A. Figure $3 \mathrm{C}$ compares $v_{0}$ extracted using this procedure with the values found independently in (8). The figure shows good agreement between the two analyses and with the viscosity expected theoretically (11).

Finally, the above hydrodynamic description is also consistent with the large negative magnetoresistance observed in our graphene devices at elevated $T$ using the standard longitudinal geometry (22). The magnetoresistance can be described accurately, without any fitting parameters (fig. S5), using the same viscosity values as found experimentally in Fig. 3C. It is interesting to note that similar magnetoresistance was reported in other high-quality 2D systems and attributed to ballistic transport affected by electron-electron interactions $(26,27)$. Only recently it has been realized that 
the anomalous negative magnetoresistance at elevated $T$ may signify the presence of a viscous flow and can also be described by the hydrodynamic approach $(15,24)$, consistent with our work (22). It would be interesting to expand studies of the Hall viscosity into the quantum Hall effect regime, which attracts considerable theory interest [see, e.g., (28-30)] but, unfortunately, no experimental procedure has so far been suggested to probe this regime.

\section{REFERENCES AND NOTES}

1. R. N. Gurzhi, Hydrodynamic effects in solids at low temperature. Sov. Phys. Usp. 11, 255-270 (1968). doi:10.1070/PU1968v011n02ABEH003815

2. M. J. M. de Jong, L. W. Molenkamp, Hydrodynamic electron flow in high-mobility wires. Phys. Rev. B 51, 13389-13402 (1995). doi:10.1103/PhysRevB.51.13389 Medline

3. A. O. Govorov, J. J. Heremans, Hydrodynamic effects in interacting Fermi electron jets. Phys. Rev. Lett. 92, 026803 (2004). do:10.1103/PhysRevLett.92.026803 Medline

4. P. J. W. Moll, P. Kushwaha, N. Nandi, B. Schmidt, A. P. Mackenzie, Evidence for hydrodynamic electron flow in $\mathrm{PdCoO}_{2}$. Science 351, 1061-1064 (2016). doi:10.1126/science.aac8385 Medline

5. J. Gooth, F. Menges, N. Kumar, V. Süß, C. Shekhar, Y. Sun, U. Drechsler, R. Zierold, C. Felser, B. Gotsmann, Thermal and electrical signatures of a hydrodynamic electron fluid in tungsten diphosphide. Nat. Commun. 9, 4093 (2018). doi:10.1038/s41467-018-06688-y Medline

6. D. A. Bandurin, I. Torre, R. Krishna Kumar, M. Ben Shalom, A. Tomadin, A. Principi, G. H. Auton, E. Khestanova, K. S. Novoselov, I. V. Grigorieva, L. A. Ponomarenko, A. K. Geim, M. Polini, Negative local resistance caused by viscous electron backflow in graphene. Science 351, 1055-1058 (2016). doi:10.1126/science.aad0201 Medline

7. D. A. Bandurin, A. V. Shytov, L. S. Levitov, R. K. Kumar, A. I. Berdyugin, M. Ben Shalom, I. V. Grigorieva, A. K. Geim, G. Falkovich, Fluidity onset in graphene. Nat. Commun. 9, 4533 (2018). doi:10.1038/s41467-018-07004-4 Medline

8. R. Krishna Kumar, D. A. Bandurin, F. M. D. Pellegrino, Y. Cao, A. Principi, H. Guo, G. H. Auton, M. Ben Shalom, L. A. Ponomarenko, G. Falkovich, K. Watanabe, T. Taniguchi, I. V. Grigorieva, L. S. Levitov, M. Polini, A. K. Geim, Superballistic flow of viscous electron fluid through graphene constrictions. Nat. Phys. 13, 1182-1185 (2017). doi:10.1038/nphys4240

9. A. Lucas, K. C. Fong, Hydrodynamics of electrons in graphene. J. Phys. Condens. Matter 30, 053001 (2018). doi:10.1088/1361-648X/aaa274 Medline

10. D. Y. H. Ho, I. Yudhistira, N. Chakraborty, S. Adam, Theoretical determination of hydrodynamic window in monolayer and bilayer graphene from scattering rates. Phys. Rev. B 97, 121404 (2018). doi:10.1103/PhysRevB.97.121404

11. A. Principi, G. Vignale, M. Carrega, M. Polini, Bulk and shear viscosities of the twodimensional electron liquid in a doped graphene sheet. Phys. Rev. B 93, 125410 (2016). doi:10.1103/PhysRevB.93.125410

12. A. Shytov, J. F. Kong, G. Falkovich, L. Levitov, Particle collisions and negative nonlocal response of ballistic electrons. Phys. Rev. Lett. 121, 176805 (2018). doi:10.1103/PhysRevLett.121.176805 Medline

13. J. Crossno, J. K. Shi, K. Wang, X. Liu, A. Harzheim, A. Lucas, S. Sachdev, P. Kim, T. Taniguchi, K. Watanabe, T. A. Ohki, K. C. Fong, Observation of the Dirac fluid and the breakdown of the Wiedemann-Franz law in graphene. Science 351, 1058-1061 (2016). doi:10.1126/science.aad0343 Medline

14. J. E. Avron, Odd viscosity. J. Stat. Phys. 92, 543-557 (1998). doi:10.1023/A:1023084404080

15. T. Scaffidi, N. Nandi, B. Schmidt, A. P. Mackenzie, J. E. Moore, Hydrodynamic electron flow and Hall viscosity. Phys. Rev. Lett. 118, 226601 (2017). doi:10.1103/PhysRevLett.118.226601 Medline

16. F. M. D. Pellegrino, I. Torre, M. Polini, Nonlocal transport and the Hall viscosity of two-dimensional hydrodynamic electron liquids. Phys. Rev. B 96, 195401 (2017). doi:10.1103/PhysRevB.96.195401

17. L. V. Delacrétaz, A. Gromov, Transport signatures of the Hall viscosity. Phys. Rev. Lett. 119, 226602 (2017). doi:10.1103/PhysRevLett.119.226602 Medline
18. I. Torre, A. Tomadin, A. K. Geim, M. Polini, Nonlocal transport and the hydrodynamic shear viscosity in graphene. Phys. Rev. B 92, 165433 (2015). doi:10.1103/PhysRevB.92.165433

19. L. Levitov, G. Falkovich, Electron viscosity, current vortices and negative nonlocal resistance in graphene. Nat. Phys. 12, 672-676 (2016). doi:10.1038/nphys3667

20. G. Falkovich, L. Levitov, Linking spatial distributions of potential and current in viscous electronics. Phys. Rev. Lett. 119, 066601 (2017). doi:10.1103/PhysRevLett.119.066601 Medline

21. F. M. D. Pellegrino, I. Torre, A. K. Geim, M. Polini, Electron hydrodynamics dilemma: Whirlpools or no whirlpools. Phys. Rev. B 94, 155414 (2016). doi:10.1103/PhysRevB.94.155414

22. Materials and methods are available as supplementary materials.

23. C. W. J. Beenakker, H. van Houten, Quantum transport in semiconductor nanostructures. Solid State Phys. 44, 1-228 (1991). doi:10.1016/S0081$1947(08) 60091-0$

24. P. S. Alekseev, Negative magnetoresistance in viscous flow of two-dimensional electrons. Phys. Rev. Lett. 117, 166601 (2016). doi:10.1103/PhysRevLett.117.166601 Medline

25. M. S. Steinberg, Viscosity of the electron gas in metals. Phys. Rev. 109, 1486-1492 (1958). doi:10.1103/PhysRev. 109.1486

26. Q. Shi, P. D. Martin, Q. A. Ebner, M. A. Zudov, L. N. Pfeiffer, K. W. West, Colossal negative magnetoresistance in a two-dimensional electron gas. Phys. Rev. B 89, 201301 (2014). doi:10.1103/PhysRevB.89.201301

27. V. T. Renard, O. A. Tkachenko, V. A. Tkachenko, T. Ota, N. Kumada, J.-C. Portal, Y Hirayama, Boundary-mediated electron-electron interactions in quantum point contacts. Phys. Rev. Lett. 100, 186801 (2008). doi:10.1103/PhysRevLett.100.186801Medline

28. J. E. Avron, R. Seiler, P. G. Zograf, Viscosity of quantum Hall fluids. Phys. Rev. Lett. 75, 697-700 (1995). doi:10.1103/PhysRevL ett.75.697 Medline

29. I. V. Tokatly, G. Vignale, Lorentz shear modulus of a two-dimensional electron gas at high magnetic field. Phys. Rev. B 76, 161305 (2007). doi:10.1103/PhysRevB.76.161305

30. F. D. M. Haldane, Geometrical description of the fractional quantum Hall effect. Phys. Rev. Lett. 107, 116801 (2011). doi:10.1103/PhysRevLett.107.116801Medline

31. A. I. Berdyuginet al., Measuring Hall viscosity of graphene's electron fluid. Zenodo (2019); doi:10.5281/zenodo.2562181.

32. L. Wang, I. Meric, P. Y. Huang, Q. Gao, Y. Gao, H. Tran, T. Taniguchi, K. Watanabe, L. M. Campos, D. A. Muller, J. Guo, P. Kim, J. Hone, K. L. Shepard, C. R. Dean, Onedimensional electrical contact to a two-dimensional material. Science 342, 614617 (2013). doi:10.1126/science.1244358 Medline

33. A. V. Kretinin, Y. Cao, J. S. Tu, G. L. Yu, R. Jalil, K. S. Novoselov, S. J. Haigh, A. Gholinia, A. Mishchenko, M. Lozada, T. Georgiou, C. R. Woods, F. Withers, P. Blake, G. Eda, A. Wirsig, C. Hucho, K. Watanabe, T. Taniguchi, A. K. Geim, R. V. Gorbachev, Electronic properties of graphene encapsulated with different twodimensional atomic crystals. Nano Lett. 14, 3270-3276 (2014) doi:10.1021/nl5006542 Medline

34. M. Ben Shalom, M. J. Zhu, V. I. Fal'ko, A. Mishchenko, A. V. Kretinin, K. S. Novoselov, C. R. Woods, K. Watanabe, T. Taniguchi, A. K. Geim, J. R. Prance, Quantum oscillations of the critical current and high-field superconducting proximity in ballistic graphene. Nat. Phys. 12, 318-322 (2016). doi: $10.1038 /$ nphys 3592

35. B. A. Braem, F. M. D. Pellegrino, A. Principi, M. Röösli, C. Gold, S. Hennel, J. V. Koski, M. Berl, W. Dietsche, W. Wegscheider, M. Polini, T. Ihn, K. Ensslin, Scanning gate microscopy in a viscous electron fluid. Phys. Rev. B 98, 241304 (2018). doi:10.1103/PhysRevB.98.241304

36. M. Beconcini, S. Valentini, R. K. Kumar, G. H. Auton, A. K. Geim, L. A. Ponomarenko, M. Polini, F. Taddei, Scaling approach to tight-binding transport in realistic graphene devices: The case of transverse magnetic focusing. Phys. Rev. B 94, 115441 (2016). doi:10.1103/PhysRevB.94.115441

37. S. Masubuchi, K. Iguchi, T. Yamaguchi, M. Onuki, M. Arai, K. Watanabe, T. Taniguchi, T. Machida, Boundary scattering in ballistic graphene. Phys. Rev. Lett. 109, 036601 (2012). doi:10.1103/PhysRevL ett.109.036601 Medline

38. A. T. Hatke, M. A. Zudov, J. L. Reno, L. N. Pfeiffer, K. W. West, Giant negative magnetoresistance in high-mobility two-dimensional electron systems. Phys. Rev. B 85, 081304 (2012). doi:10.1103/PhysRevB.85.081304 
39. M. Y. Melnikov, J. P. Kotthaus, V. Pellegrini, L. Sorba, G. Biasiol, V. S. Khrapai, Influence of e-e scattering on the temperature dependence of the resistance of a classical ballistic point contact in a two-dimensional electron system. Phys. Rev. B 86, 075425 (2012). doi:10.1103/PhysRevB.86.075425

40. G. M. Gusev, A. D. Levin, E. V. Levinson, A. K. Bakarov, Viscous electron flow in mesoscopic two-dimensional electron gas. AIP Adv. 8, 025318 (2018). doi:10.1063/1.5020763

\section{ACKNOWLEDGMENTS}

Funding: This work was supported by the European Research Council, the Graphene Flagship and Lloyd's Register Foundation. D.A.B. acknowledges the financial support from Leverhulme Trust, and R.K.K. from the EPSRC. Author contributions:D.A.B. and A.K.G. designed and supervised the project. A.I.B. and D.A.B. performed electrical measurements. S.G.X. and M.B.S. fabricated devices. A.I.B. and D.A.B. analyzed the data with input from R.K.K. and A.K.G.; F.M.D.P., A.P., I.T. and M.P. provided theoretical support. T.T. and K.W. provided hBN crystals. D.A.B. and A.I.B. wrote the manuscript with help from A.K.G. All the authors contributed to discussions. Competing interests: The authors declare no competing interests. Data and materials availability: The data reported in this work are archived at Zenodo (31).

\section{SUPPLEMENTARY MATERIALS}

www.sciencemag.org/cgi/content/full/science.aau0685/DC1

Supplementary Text

Figs. S1 to S6

References (32-40)

4 May 2018; accepted 19 February 2019

Published online 28 February 2019

10.1126/science.aau0685 


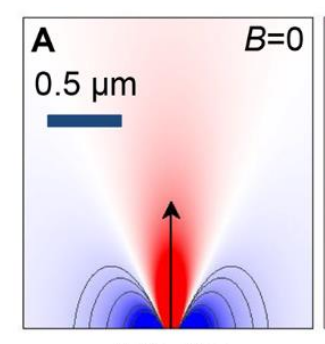

Injector

E

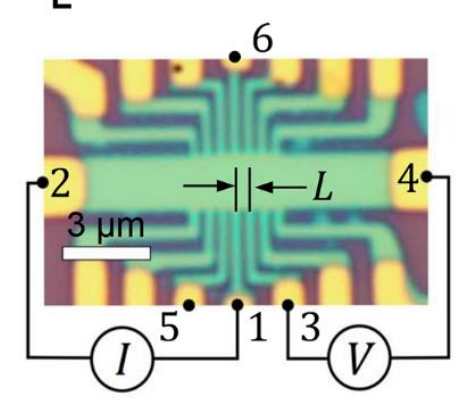

B $\quad B \neq 0$
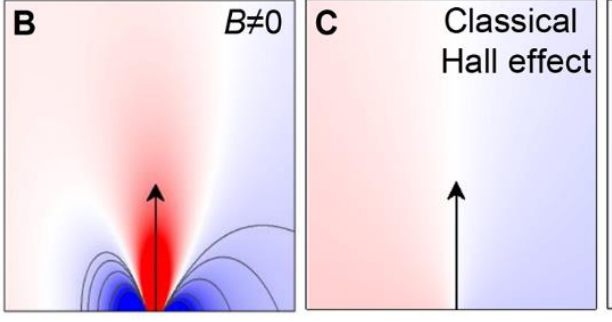

$\mathbf{F}$

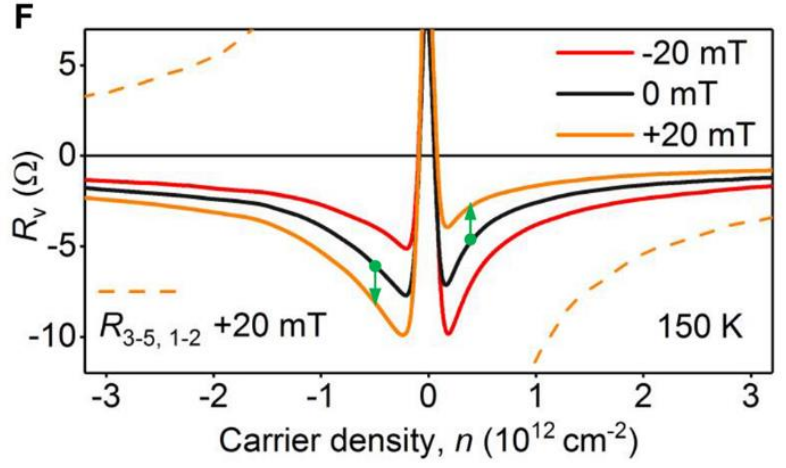

Fig. 1. Effect of magnetic field on viscous electron flow. ( $A$ and $B$ ) Electric potential distribution $\phi(r)$ expected in graphene's electron fluid near a current injector in zero $B$ and $50 \mathrm{mT}$, respectively. The calculations were based on Eq. C15-C16 of (16) using characteristic $v_{0}=0.1 \mathrm{~m}^{2} / \mathrm{s}$, found in our experiments below, $n$ $=2 \times 10^{12} \mathrm{~cm}^{-2}$ and $\tau=2 \mathrm{ps}$. Solid curves: Equipotentials. (C) Contribution from the ordinary Hall effect toward the map in (B). (D) Contribution that comes from $v_{H}$. Color scale in (A-D): dark-blue to dark-red, -2.5 to +2.5 of the potential induced by the ordinary Hall effect in (C). (E) Optical micrograph of one of our devices, along with the schematic of the vicinity geometry from which $R_{v}$ is obtained. (F) Examples of the vicinity resistance for different $B$ (solid curves); $L \approx 1 \mu \mathrm{m}$. Dashed: Local Hall resistance measured using voltage probes 3 and 5 close to the current injector (22). 

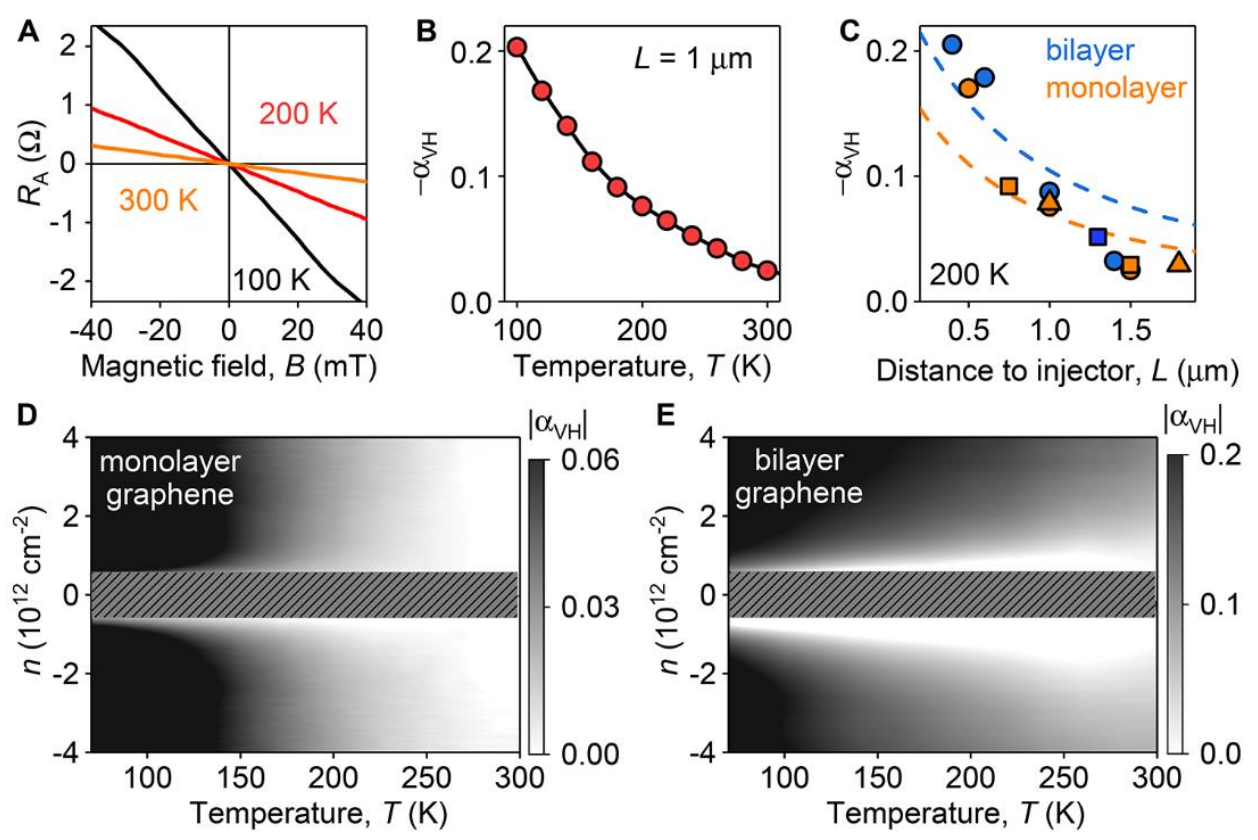

Fig. 2. Viscous Hall effect. (A) $R_{A}(B)$ for one of our MLG devices at $L \approx 1 \mu \mathrm{m}$ for three different temperatures. (B) The dimensionless viscous coefficient $\alpha_{\mathrm{VH}}(T) \quad$ (symbols). For reproducibility, see fig. S2C. (C) $\alpha_{V H}(L)$ found for 5 different devices indicated by different symbols whereas the color refers to $M L G$ and BLG. $n=2 \times 10^{12} \mathrm{~cm}^{-2}$ for $(A-C)$, which corresponds to the Fermi energy of $\sim 165 \mathrm{meV}$ and $\sim 70 \mathrm{meV}$ for MLG and BLG, respectively. Dashed lines in $(C)$ : Dependences from Eq. 2 with no fitting parameters: $\sigma_{0}(T)$ is determined as described in Section 1 of (22) and $v_{0}(T)$ is taken from experiment (6). (D and E) Maps of $\left|\alpha_{\mathrm{VH}}\right|$ in MLG and BLG devices for $L \approx 1.5 \mu \mathrm{m}$ and $0.7 \mu \mathrm{m}$, respectively; $B=40 \mathrm{mT}$. Shaded areas: Omitted analysis because the cyclotron diameter becomes comparable to the device width (23). 

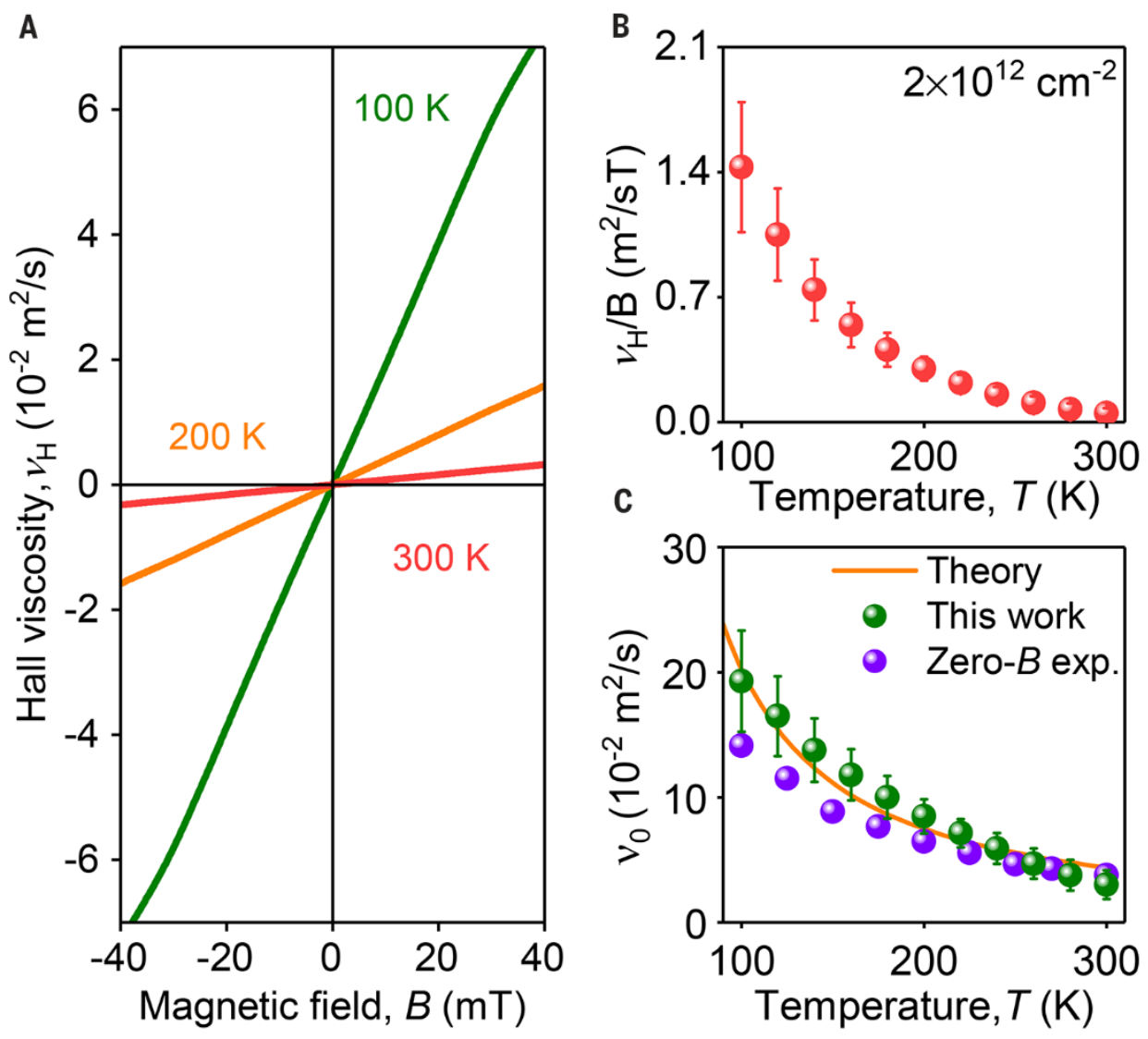

Fig. 3. Hall viscosity in graphene. (A) Examples of $V_{H}$ extracted using data from Fig. 2A. (B) $v_{H} / B$ as a function of $T$. (C) Zero-field viscosity $v_{0}$ extracted from our $R_{\mathrm{A}}(B)$ measurements (green). Solid curve: Theory (11). Purple symbols: Previous experiments (8). For all the panels: MLG at $n$ $=2 \times 10^{12} \mathrm{~cm}^{-2}$. No fitting parameters were used for the theory curve in (C). Error bars in (B) and (C) represent the scatter for measurements using different $L$. The notable increase of the error below 150 $\mathrm{K}$ indicates that the electron system starts exiting the hydrodynamic regime $(7,12)$. 